\title{
Influencia emocional en árbitros de fútbol aplicando tecnologías de sensores
}

\section{Emotional influence on soccer referees applying sensor technologies}

\author{
Luis Arturo Espín Pazmiño ${ }^{1,2}$ https://orcid.org/0000-0002-1663-2489, \\ Margareth Priscilla Enderica Malo² \\ ${ }^{1}$ Universidad Nacional de la Plata - Facultad de Informática, La Plata, Argentina \\ luis.espinp@ug.edu.ec \\ ${ }^{2}$ Universidad de Guayaquil - Facultad de Ciencias Matemáticas y Físicas, \\ Guayaquil, Ecuador \\ margareth.endericameug.edu.ec
}

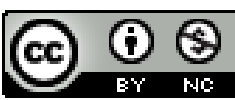

Esta obra está bajo una licencia internacional Creative Commons Atribución-NoComercial 4.0. $\begin{array}{ll}\text { Enviado: } & 2021 / 07 / 11 \\ \text { Aceptado: } & 2021 / 09 / 28 \\ \text { Publicado: } & 2021 / 11 / 30\end{array}$

\section{Resumen}

En el ámbito deportivo, las decisiones arbitrales siempre han estado bajo la lupa de los deportistas, directivos y espectadores, por tal motivo se mantiene en constante evaluación y entrenamiento el tema de reducir el error. Es necesario exponer que la toma de decisiones se puede generar bajo la influencia directa de las emociones, puesto que el proceso de respuestas emocionales es inconsciente y pueden interferir durante el desarrollo de diversas actividades, en este caso, a las relacionadas con el arbitraje. Por ello, se genera un prototipo de sensores en función a los parámetros fisiológicos de la temperatura corporal y la frecuencia cardiaca como patrones de expresión de diversos estados emocionales de este grupo de deportistas, y a base de la representación del modelo de afectividad positiva y negativa, se identifica el estado o cambios de estados emocionales durante la ejecución de un partido. Luego, con uso del material audiovisual se realiza, con criterio de expertos, el análisis de toma de decisiones de los participantes en estas pruebas. Los árbitros participantes, en las pruebas de campo, se les registra con mayor frecuencia dos estados relacionados con valencia positiva y solo un estado de valencia negativa, sin embargo, en la mayoría de los casos, en el análisis de datos se observa que la toma de decisiones se alinea correctamente con el reglamento.

Palabras clave: estados emocionales, frecuencia cardiaca, parámetros fisiológicos. 


\begin{abstract}
In the sports field, referee decisions have always been under the scrutiny of athletes, managers, and spectators. For this reason, there is constant assessment and training to reduce error. It is necessary to state that decision-making can be generated under the direct influence of emotions, since the process of emotional responses is unconscious and can interfere during the development of different activities: in this case those related to refereeing. A prototype of sensors was generated based on the physiological parameters of body temperature and heart rate as expression patterns of various emotional states of the athlete based on the representation of the model of positive and negative affectivity, the state or changes of states were identified during the execution of the match. Then, with the use of audio-visual material, an analysis of the decision-making of the participants in these tests was carried out by experts on this criterion. Two states related to positive valence and only one negative valence state was recorded more frequently in the field tests of the participating referees. However, in most cases, in the data analysis it was observed that the decision-making was correctly aligned with the sport regulations.
\end{abstract}

Keywords: emotional states, heart rate, physiological parameters.

\title{
Introducción
}

En la actualidad, son pocos los desarrollos tecnológicos destinados a la medición fisiológica de las emociones en el deporte. Por ello, esta investigación busca promover la implementación de las tecnologías aplicadas al deporte, relacionando estudios de las ramas de la medicina y psicología. Es así que se consigue el desarrollo de un prototipo, tomando en cuenta las bases científicas de las distintas áreas, considerando específicamente la representación del modelo de afectividad positiva y negativa (Watson y Tellegen, 1985). De acuerdo con la propuesta de ejes ortogonales (Yik, et al., 1999) (Figura 1) y con la finalidad del constante uso de las tecnologías para mejorar desempeños y aprendizajes en el ámbito deportivo, en este caso, se estudia la labor arbitral en los partidos de fútbol. Además, la utilidad de esta herramienta tecnológica permite poder realizar investigaciones de campo, no solo en el ámbito del arbitraje, sino también, en los diferentes aspectos de los deportes donde el estado emocional pueda generar un punto de inflexión en la toma de decisiones.

\section{Figura 1}

Representación del modelo de afectividad

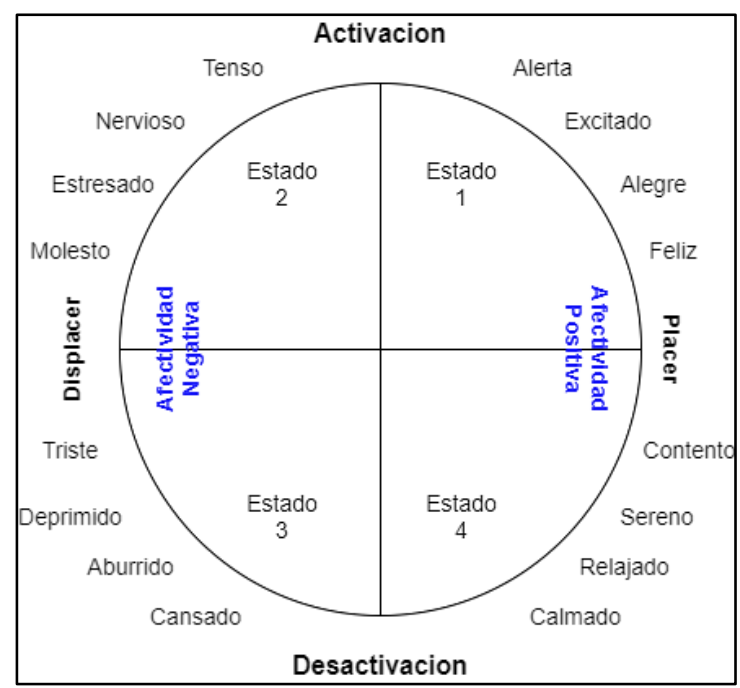


Dado el escaso hallazgo de antecedentes investigativos en el país sobre la influencia emocional ante la toma de decisiones de los árbitros de fútbol, desde el punto de vista de esta investigación, se considera fundamental el uso de nuevas tecnologías para colaboración de futuros estudios deportivos. Con el fin de permitir la recolección de datos de las diferentes variables biométricas y, con ello, generar un apoyo en el trabajo de analítica deportiva, centrada en el rendimiento deportivo y el desempeño de los atletas.

En la mayoría de las ocasiones, las decisiones tomadas por un árbitro de fútbol son siempre cuestionadas, a través de la opinión de los espectadores del evento y de los analistas deportivos. Las decisiones tomadas por los árbitros pueden llegar a marcar el rumbo de la definición del partido, lo que hace que esta actividad de arbitraje sea indispensable para un adecuado desarrollo del juego (Escobar, et al., 2020).

La emoción es un estado afectivo intenso de breve duración que va acompañado de respuestas neurales y hormonales. Cuando se experimenta una emoción, se activa el sistema nervioso autónomo, en el que interactúan la rama simpática (donde se viven las emociones intensas, se eleva el ritmo cardiaco, se tensan los músculos y aumenta la sudoración) y parasimpática (se viven las emociones de relajación y descanso, disminuye la frecuencia cardiaca y se relajan los músculos) (Ortiz, 2010).

Las emociones son reacciones que se desencadenan en tres niveles diferentes: la experiencia emocional, los cambios corporales y la conducta. Dichas reacciones se vivencian como una fuerte conmoción del estado de ánimo (estado afectivo), suelen tener un marcado acento placentero o desagradable y van acompañadas por la percepción de cambios orgánicos, o corporales, a veces muy intensos (experiencia emocional). Los cambios corporales de la emoción se caracterizan por una elevada activación fisiológica, especialmente del sistema nervioso autónomo (sudoración excesiva, aumento de la tasa cardiaca, etc.) y del sistema nervioso somático (tensión muscular), aunque también se activan otros sistemas (hormonal, inmune, etc.) (Vindel, 2010).

Los árbitros deben estar formados en tácticas de trabajo que permitan una optimización de sus funciones. Para ello, los planes de trabajo planificados deben contar con formación en el manejo adecuado de la atención, preparación en la activación para el control de impulsos, el desarrollo del conocimiento de las diversas motivaciones que surgen. Se suma el manejo de canales de comunicación desde sí mismo hasta la relación con su contexto, el fortalecimiento de la autoestima y autoconfianza utilizando un modelo de jerarquización que permita al árbitro formarse en el autocontrol en un primer nivel. Además, tomar decisiones claras y ajustadas a un partido con la rapidez que éste lo requiere. Un segundo nivel más básico debe estar encaminado a la necesidad de formar en el manejo de la atención; y un tercer nivel de formación en el manejo de las motivaciones. (Ortega, et al., 2014)

En su tesis de Maestría "El desarrollo emocional en el desempeño conductual de los árbitros profesionales de fútbol del cantón de Ambato", se enfatiza el rol importante que desarrollan los árbitros y la formación del estado psicológico emocional - interconductual, que se considera debe ir de la mano con la preparación física. Menciona el equilibrio que debe existir entre la educación y la inteligencia emocional del árbitro. El estudio se realiza a un grupo de 47 sujetos ligados a la actividad arbitral, a quienes se les realiza una prueba donde se obtiene valores de: autoconciencia, control de las emociones, automotivación, relacionarse bien, asesoría emocional, donde se concluye que el trabajo de un árbitro es muy complejo y que requiere de una completa preparación estratégica emocional para su óptimo rendimiento psicológico (Fonseca Mora, 2017). 
El proyecto, "Desarrollo de técnicas de detección automática de estado de ánimo mediante redes de sensores inalámbricas", se divide en diferentes módulos donde se realizan pruebas en las que se estimula al usuario a sentir determinadas emociones y mediante el uso de herramientas tecnológicas y diagramaciones -usando dos dispositivos- capturaron las señales biométricas entre ellas ECG y temperatura, comparándolos entre sí mediante los histogramas y relacionándolas con la emoción inducida (Cisneros, et al., 2013).

La FCmed. ${ }^{1}$ lograda por los árbitros en un estudio que se desarrolló durante el Campeonato del Mundo de categoría sub-17 organizado por la FIFA y que se disputó en Finlandia en agosto del año 2003 fue de $157 \pm 4 \mathrm{p} / \mathrm{min}$, lo que suponía el $83 \pm 1 \%$ de la FCmáx. $^{2}$ de los jueces (Mallo, García, y Navarro, 2007). La FCpico ${ }^{3}$ (bpm) alcanzada en un partido por un árbitro central es de 183,10 \pm 10,39, la FCmed 160.59 $\pm 12,41$ (Castillo, et al., 2016).

En un mundo tan competitivo como es el del deporte, cualquier detalle por mínimo que sea, marca una gran diferencia. Los datos que ofrecen los diferentes sensores y otros medios, aportan una ventaja competitiva extra que puede ser diferencial. La tecnología ha alcanzado la industria del deporte, y ha impactado en los resultados de los equipos y deportistas, debido a las mejoras en las técnicas, a la aplicación de nuevos métodos de entrenamiento y al análisis predictivo de los datos (Fava, et al., 2020).

\section{Instrumentos}

\section{Materiales y Métodos}

Para este estudio se utilizan sensores inalámbricos integrados a un chaleco de vestir para el árbitro. El prototipo se desarrolla con un nodo de sensores cuya función es medir variables fisiológicas específicas como la temperatura (sensor LM35) y la frecuencia cardiaca (AD8232) (Maier, et al., 2017), conectados a un módulo inalámbrico (ESP32), encargado de enviar esa información recabada a un nodo base puente IEEE 802.15.4/802.11, todo esto alimentado por un banco de baterías.

\section{Figura 2}

Interfaz gráfica - Estado emocional 4

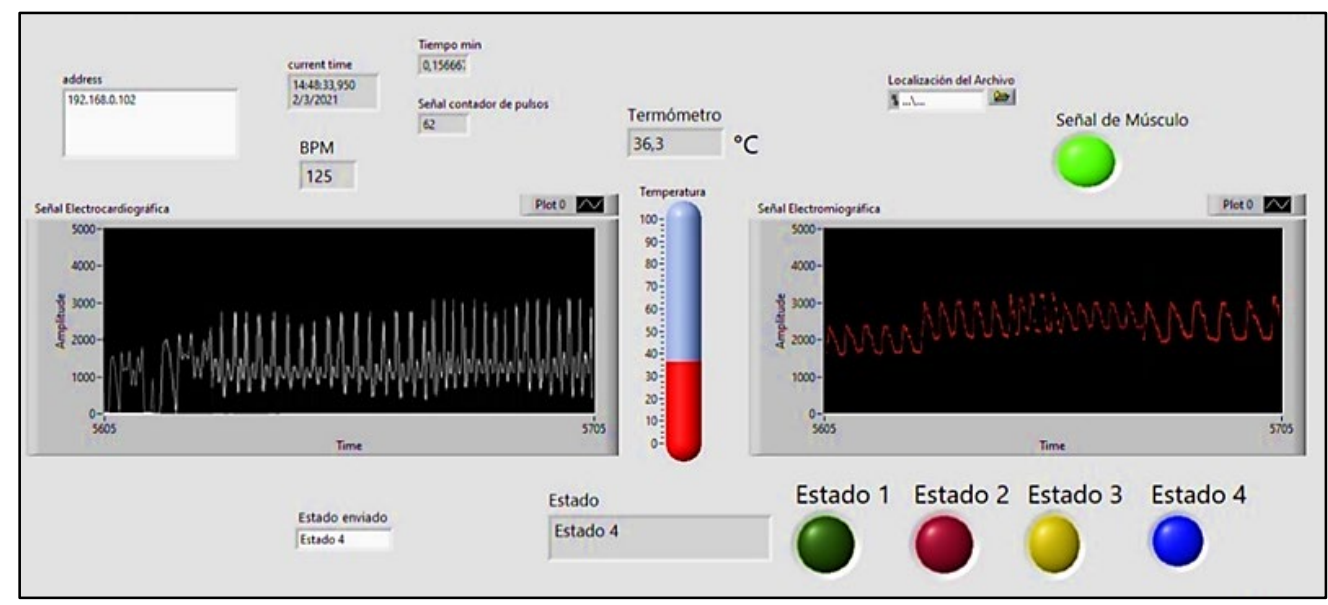

\footnotetext{
${ }^{1}$ Frecuencia cardiaca media

${ }^{2}$ Frecuencia cardiaca máxima.

${ }^{3}$ Frecuencia cardiaca pico.
} 
El objetivo es captar las respuestas sensoriales del organismo durante el desarrollo del juego de fútbol. Los datos obtenidos se registran y se traducen a través de un dispositivo ESP32 con la ayuda del programa LABVIEW (Figura 2), que permite generar la estadística necesaria de los datos fisiológicos considerando los rangos preestablecidos para el respectivo análisis de los estados emocionales del sujeto durante el transcurso del juego.

\section{Método - Participantes}

Para la ejecución del presente estudio se considera a la participación de los integrantes de la Asociación de árbitros de fútbol del Guayas que tiene registrados aproximadamente 130 profesionales del arbitraje, quienes dirigen diferentes categorías del fútbol profesional y amateur del país. Con el fin de tener un panorama concreto referente a las actividades que realizan los árbitros en su preparación en el área de inteligencia emocional y psicológica. Se aplica, además, una encuesta descriptiva para cumplir los objetivos planteados, y se realizan pruebas de campo a 8 árbitros (31,4 \pm años) que colaboran con esta investigación, en encuentros de fútbol y mini-fútbol amistosos, entre amateur e infantiles, durante un tiempo de prueba definido de 30 minutos para todos los participantes. Antes del inicio de las pruebas de campo se explica a los ocho árbitros la naturaleza de la investigación y se obtiene el respectivo consentimiento de todos los involucrados (Figura 3).

\section{Figura 3}

Participante con el prototipo

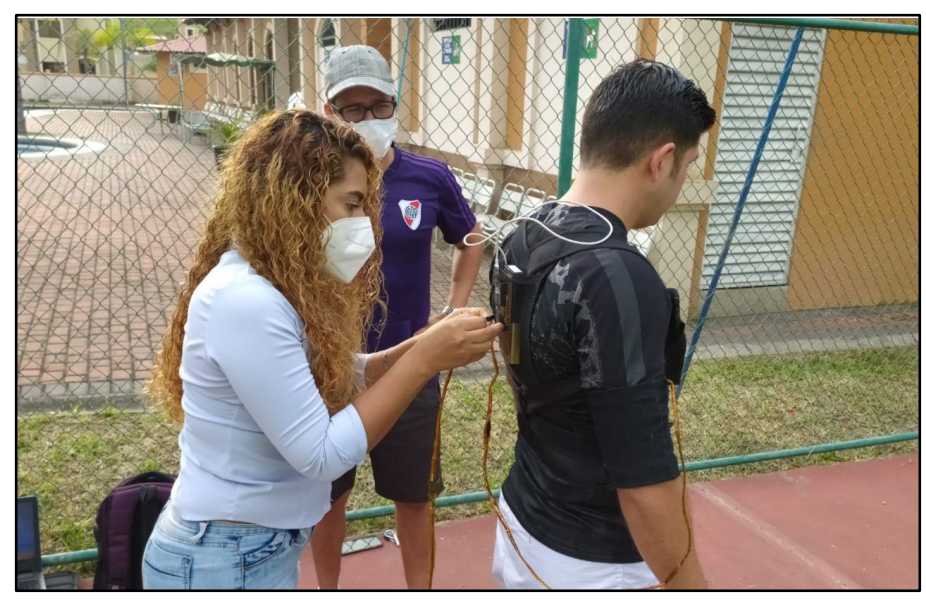

\section{Metodología}

Se definió un estudio de tipo cualitativo-cuantitativo, puesto que en el proceso de este trabajo se manipularon datos no paramétricos que ayudaron a cumplir con los objetivos planteados y su registro con la finalidad de detectar la expresión biológica de las emociones.

Se utilizó un diseño mixto: (i) investigación de antecedentes a través de la recopilación de información de las fuentes primarias y secundarias enmarcados en el estudio de caso relacionadas con la influencia de las emociones durante la toma de decisiones arbitrales. Se planteó esta revisión bibliográfica con base en las manifestaciones fisiológicas del ser humano como reacción ante las emociones en determinadas situaciones y se las vinculó con la acciones o decisiones tomadas en la actividad del arbitraje. Y (ii) para la monitorización de los estados de ánimo se utilizó un diseño cuasi experimental con un grupo experimental para toma de lecturas analógicas de la frecuencia cardiaca y temperatura del usuario, previo y durante su actividad de arbitraje para su posterior interpretación. 


\section{Tipo de estudio}

Se aplicó como técnica la recolección de datos y su posterior observación para llegar a un análisis de estudio de un tema poco explorado, para futuras investigaciones o proyectos tecnológicos. El método de recolección de datos biométricos estuvo basado en el uso de la plataforma de código libre, Arduino, que brindaba datos cuantitativos para la interpretación de los parámetros de los estados emocionales del individuo en la acción de arbitraje. El método investigativo aplicado en este proyecto se desarrolló mediante técnicas de comparación de la reacción fisiológica relacionadas con las emociones, debido a la respuesta concatenada del sistema simpático y parasimpático.

\section{Procedimiento}

Los datos comparados son de tipo primario, puesto que se tomaron en tiempo real mediante sensores de magnitud física que fueron presentados en una interfaz gráfica. Estos datos cuantitativos obtenidos por los sensores fueron almacenados en una base de datos para su posterior consulta, visualización y análisis considerando los siguientes procedimientos:

- Registro de observación: Se realizó vídeo análisis con expertos del arbitraje observando el material audiovisual registrado de los partidos.

- Prototipo de registro de parámetros físiológicos: Desarrollado con el objetivo de establecer la toma de las variables biométricas durante la ejecución de la tarea del arbitraje.

Los datos analizados fueron:

\section{Frecuencia cardiaca}

Se registró el rendimiento cardiaco a lo largo del desarrollo de la prueba a través de un receptor ECG. La respuesta de la frecuencia cardiaca durante un partido de fútbol se ha utilizado tradicionalmente como un indicador indirecto del gasto energético que supone la actividad. Los valores de frecuencia cardiaca media (FCmed) registrados en los árbitros durante los partidos se sitúan en el rango de las 153 a las 165 p/min. (Mallo, et al., 2007).

\section{Temperatura corporal}

Se registró la variación de la temperatura corporal durante cada prueba a través del sensor de temperatura. La temperatura corporal normal cambia según la persona, la edad, las actividades y el momento del día. La temperatura corporal normal promedio aceptada es generalmente de $98.6^{\circ} \mathrm{F}\left(37^{\circ} \mathrm{C}\right)$. Algunos estudios han mostrado que la temperatura corporal 'normal' puede tener un amplio rango que va desde los $97^{\circ} \mathrm{F}\left(36.1^{\circ} \mathrm{C}\right)$ hasta los $99^{\circ} \mathrm{F}\left(37.2^{\circ} \mathrm{C}\right)$. (MedlinePlus, 2019)

Con el análisis de los datos mencionados junto con otras investigaciones médicas, tomadas como base, se realizó la Tabla 1 comparativa de rangos fisiológicos establecidos de los cuatro estados subjetivos que se utilizaron para la programación del aplicativo en las pruebas de campo. 
Tabla 1

Variables operativas de estados emocionales

\begin{tabular}{|c|c|c|c|c|}
\hline \multicolumn{5}{|c|}{ Rango de valores para pruebas } \\
\hline $\begin{array}{c}\text { ESTADOS } \\
\text { EMOCIONALES }\end{array}$ & $\begin{array}{c}\text { INDICADOR } \\
\text { FRECUENCIA } \\
\text { CARDIACA }\end{array}$ & $\begin{array}{c}\text { INDICADOR } \\
\text { TEMPERATURA }\end{array}$ & $\begin{array}{c}\text { FRECUENCIA } \\
\text { CARDIACA } \\
(\mathbf{p m})^{4}\end{array}$ & $\begin{array}{c}\text { TEMPERATURA } \\
\left({ }^{\circ} \text { C) }\right.\end{array}$ \\
\hline EE 1 & Alta 1 & Normal 1 & $150-175$ & $36,5-37,6$ \\
\hline EE 2 & Alta 2 & Alta & $176+$ & $37,1-40$ \\
\hline EE 3 & Baja & Baja & $100-119$ & $35-36$ \\
\hline EE 4 & Normal & Normal 2 & $120-149$ & $36,1-37,2$ \\
\hline
\end{tabular}

\section{A base del análisis realizado en la entrevista}

\section{Resultados y Discusión}

La preparación arbitral genera la educación emocional adecuada para evitar que el estado emocional afecte a las decisiones tomadas en cancha durante la ejecución de un partido de fútbol. La autopercepción emocional manifiesta en los árbitros participantes, hace referencia a estados emocionales del tipo 1 y 4 durante el transcurso de un juego de fútbol y la toma de decisiones cuando se comenten faltas antirreglamentarias.

Además, se obtienen los siguientes datos relevantes:

El 96.6\% de los encuestados, de acuerdo con su experiencia consideran que la FC y la TC, sí son una manifestación ante la presencia de un estado emocional.

El 94.3\% de los árbitros de la asociación de árbitros de fútbol del Guayas están de acuerdo en que de una u otra forma los estados emocionales tienen influencia en la toma de decisiones en un partido de fútbol.

El $93.3 \%$ de la muestra considera importante el manejo de la inteligencia emocional como parte de la preparación en la etapa instruccional de los árbitros de fútbol.

El $95.5 \%$ indica que está totalmente de acuerdo con el uso de la tecnología para preparación de los árbitros de fútbol.

El $95.5 \%$, manifiesta que resulta conveniente usar un prototipo con tecnología inalámbrica que permita indicar el estado emocional en tiempo real durante un partido de fútbol.

\section{Con base en los resultados obtenidos en las pruebas}

Los profesionales del arbitraje son conscientes de la influencia de los estados emocionales que puedan presentar y el nivel de interferencia en una toma de decisiones cuando se encuentran realizando su labor.

Se identifican sentimientos relacionados con el estado emocional tipo 1 y 4 de la tabla de estados emocionales desarrollada para este proyecto, que pertenecen al grupo de emociones de valencia positiva y de activación-desactivación respectivamente. Por lo tanto, se puede

\footnotetext{
${ }^{4}$ Latidos por minuto.
} 
inferir que su estado emocional es el ideal para el desarrollo de la actividad y no afectaría en la toma de decisiones, independientemente de la aplicación del reglamento.

\section{Del análisis de los datos de registros biométricos}

No existen evidencias sólidas que permitan establecer la relación entre la identificación de los estados emocionales positivos y el correcto uso del reglamento durante el transcurso de las pruebas registradas en este proyecto.

En la Tabla 2, considerando que el tiempo de la recolección de datos fue de 30 minutos, se puede observar el registro de los estados por frecuencias (frecuencia = variación por minuto) de las pruebas de campo realizadas a los 8 árbitros de fútbol participantes, identificando que la mayor distribución de los resultados se encuentra en los estados 1 y 4 , solo en 3 participantes presentan más de dos estados y en ningún participante se identifica presencia de los 4 estados.

Tabla 2

Resultados del registro por frecuencia

\begin{tabular}{|c|c|c|c|c|}
\hline \multicolumn{5}{|c|}{ Resultados de registro de estados por frecuencias } \\
\hline Participante & Estado 1 & Estado 2 & Estado 3 & Estado 4 \\
\hline $\mathbf{1}$ & 12 & 0 & 0 & 18 \\
\hline $\mathbf{2}$ & 16 & 0 & 0 & 14 \\
\hline $\mathbf{3}$ & 16 & 5 & 0 & 9 \\
\hline $\mathbf{4}$ & 7 & 0 & 0 & 23 \\
\hline $\mathbf{5}$ & 9 & 0 & 2 & 19 \\
\hline $\mathbf{6}$ & 26 & 1 & 0 & 3 \\
\hline $\mathbf{7}$ & 22 & 0 & 0 & 8 \\
\hline $\mathbf{8}$ & 14 & 0 & 0 & 16 \\
\hline
\end{tabular}

Los estados emocionales más registrados en frecuencia se relacionan con los tipos 1 y 4, es decir, el comportamiento emocional en los árbitros de fútbol se encuentra estable durante la ejecución del partido, como se muestra en la Tabla 3.

Tabla 3

Matriz de pruebas

\begin{tabular}{|c|c|c|c|c|c|c|c|}
\hline \multicolumn{8}{|c|}{ MATRIZ DE PRUEBAS REALIZADAS } \\
\hline ฟै & 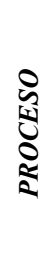 & 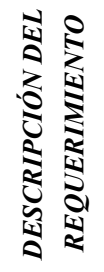 & 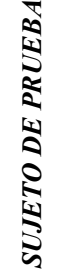 & 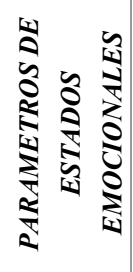 & 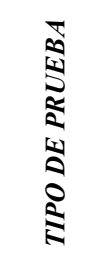 & 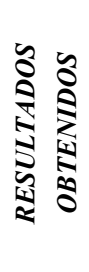 & 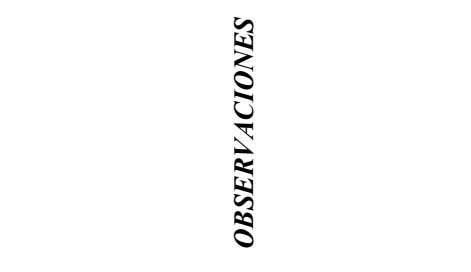 \\
\hline 1 & 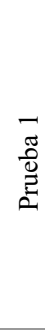 & & & $\begin{array}{l}\text { EE } 1 \\
\text { EE } 2 \\
\text { EE } 3 \\
\text { EE } 4\end{array}$ & $\begin{array}{c}\text { De } \\
\text { campo }\end{array}$ & $\begin{array}{l}\text { EE } 1 \\
\text { EE } 4\end{array}$ & $\begin{array}{l}\text { Esta prueba se la realizó a un árbitro } \\
\text { profesional. } \\
\text { A los } 16 \text { minutos, el árbitro mostro tarjeta } \\
\text { roja a un jugador, durante este suceso el } \\
\text { aplicativo registró estado } 1 \text {. } \\
\text { El experto señaló que las acciones } \\
\text { tomadas por el árbitro estuvieron } \\
\text { acertadas. }\end{array}$ \\
\hline
\end{tabular}




\begin{tabular}{|c|c|c|c|c|c|c|}
\hline 2 & 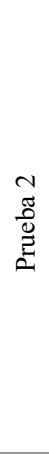 & $\begin{array}{l}\text { Mediante el uso de la } \\
\text { herramienta de } \\
\text { recolección de datos, } \\
\text { se obtuvieron los } \\
\text { parámetros fisiológicos } \\
\text { (Frecuencia cardiaca y } \\
\text { temperatura) } \\
\text { segmentados por el } \\
\text { sistema en un estado. }\end{array}$ & $\begin{array}{l}\text { Árbitros de fútbol } \\
\text { profesionales y } \\
\text { amateur utilizando } \\
\text { el chaleco prototipo, } \\
\text { dirigiendo cada } \\
\text { participante durante } \\
30 \text { minutos, partidos } \\
\text { amistosos amateur e } \\
\text { infantiles de fútbol } \\
\text { o mini-fútbol. }\end{array}$ & $\begin{array}{l}\mathrm{EE} 1 \\
\mathrm{EE} 2 \\
\mathrm{EE} 3 \\
\mathrm{EE} 4\end{array}$ & $\begin{array}{l}\text { EE } 1 \\
\text { EE } 4\end{array}$ & $\begin{array}{l}\text { Esta prueba se la realizó a un árbitro } \\
\text { profesional. } \\
\text { A los } 12 \text { minutos, el árbitro mostró una } \\
\text { tarjeta amarilla, durante este suceso se } \\
\text { encontraba en el estado } 4 \text {, se mostró } \\
\text { material audiovisual del encuentro al } \\
\text { experto, quien indicó que la decisión } \\
\text { arbitral en este caso se debe más a la } \\
\text { percepción de la intención del jugador en } \\
\text { cancha, pero a pesar de esto califica } \\
\text { como correcto el actuar del árbitro. }\end{array}$ \\
\hline 3 & 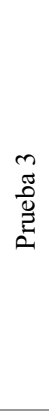 & & & $\begin{array}{l}\mathrm{EE} 1 \\
\mathrm{EE} 2 \\
\mathrm{EE} 3 \\
\mathrm{EE} 4\end{array}$ & $\begin{array}{l}\text { EE } 1 \\
\text { EE } 2 \\
\text { EE } 4\end{array}$ & $\begin{array}{l}\text { Esta prueba se la realizó a un árbitro } \\
\text { amateur. } \\
\text { A los } 20 \text { minutos existió una mano dentro } \\
\text { del área, de esta jugada se armó una } \\
\text { disputa entre los jugadores, el árbitro } \\
\text { dictaminó saque de esquina. Durante } \\
\text { este suceso, el árbitro se registró } 5 \\
\text { minutos en el Estado } 2 \text {. } \\
\text { El experto señaló que esta acción debió } \\
\text { ser sancionada como penal. }\end{array}$ \\
\hline 4 & 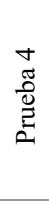 & & & $\begin{array}{l}\mathrm{EE} 1 \\
\mathrm{EE} 2 \\
\mathrm{EE} 3 \\
\mathrm{EE} 4\end{array}$ & $\begin{array}{l}\text { EE } 1 \\
\text { EE } 4\end{array}$ & $\begin{array}{l}\text { Esta prueba se la realizó a un árbitro } \\
\text { amateur. } \\
\text { El experto señaló que las acciones } \\
\text { tomadas por el árbitro estuvieron } \\
\text { acertadas. }\end{array}$ \\
\hline 5 & 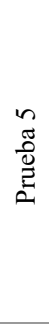 & & & $\begin{array}{l}\mathrm{EE} 1 \\
\mathrm{EE} 2 \\
\mathrm{EE} 3 \\
\mathrm{EE} 4\end{array}$ & $\begin{array}{l}\text { EE } 1 \\
\text { EE } 3 \\
\text { EE } 4\end{array}$ & $\begin{array}{l}\text { Esta prueba se la realizó a un árbitro } \\
\text { profesional. } \\
\text { En este caso se identificó en el registro de } \\
\text { frecuencias estado } 3 \text { durante } 2 \text { minutos al } \\
\text { inicio del encuentro, sin embargo, en la } \\
\text { revisión del vídeo, el experto señaló a las } \\
\text { acciones tomadas por el árbitro, como } \\
\text { acertadas. }\end{array}$ \\
\hline 6 & 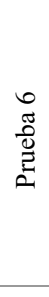 & & & $\begin{array}{l}\mathrm{EE} 1 \\
\mathrm{EE} 2 \\
\mathrm{EE} 3 \\
\mathrm{EE} 4\end{array}$ & $\begin{array}{l}\text { EE } 1 \\
\text { EE } 2 \\
\text { EE } 4\end{array}$ & $\begin{array}{l}\text { Esta prueba se la realizó a un árbitro } \\
\text { amateur. } \\
\text { En este caso se identificó en el registro de } \\
\text { frecuencias estado } 2 \text { durante } 1 \text { minuto, } \\
\text { sin embargo, en la revisión del vídeo, el } \\
\text { experto señaló a las acciones tomadas por } \\
\text { el árbitro, como acertadas. }\end{array}$ \\
\hline 7 & 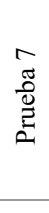 & & & $\begin{array}{l}\mathrm{EE} 1 \\
\mathrm{EE} 2 \\
\mathrm{EE} 3 \\
\mathrm{EE} 4\end{array}$ & $\begin{array}{l}\text { EE } 1 \\
\text { EE } 4\end{array}$ & $\begin{array}{l}\text { Esta prueba se la realizó a un árbitro } \\
\text { profesional. } \\
\text { El experto señaló que las acciones } \\
\text { tomadas por el árbitro estuvieron } \\
\text { acertadas. }\end{array}$ \\
\hline 8 & 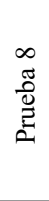 & & & $\begin{array}{l}\mathrm{EE} 1 \\
\mathrm{EE} 2 \\
\mathrm{EE} 3 \\
\mathrm{EE} 4\end{array}$ & $\begin{array}{l}\text { EE } 1 \\
\text { EE } 4\end{array}$ & $\begin{array}{l}\text { Esta prueba se la realizó a un árbitro } \\
\text { profesional. } \\
\text { El experto señaló que las acciones } \\
\text { tomadas por el árbitro estuvieron } \\
\text { acertadas. }\end{array}$ \\
\hline
\end{tabular}

El comportamiento de los valores fisiológicos de frecuencia cardiaca y temperatura corporal de cada participante en las pruebas de campo, se muestran en la Figura 4 y Figura 5, respectivamente. 


\section{Figura 4}

Registro de la frecuencia cardiaca (lpm) (Y) de cada árbitro participante en las pruebas durante 30 minutos $(X)$ en un gráfico de dispersión

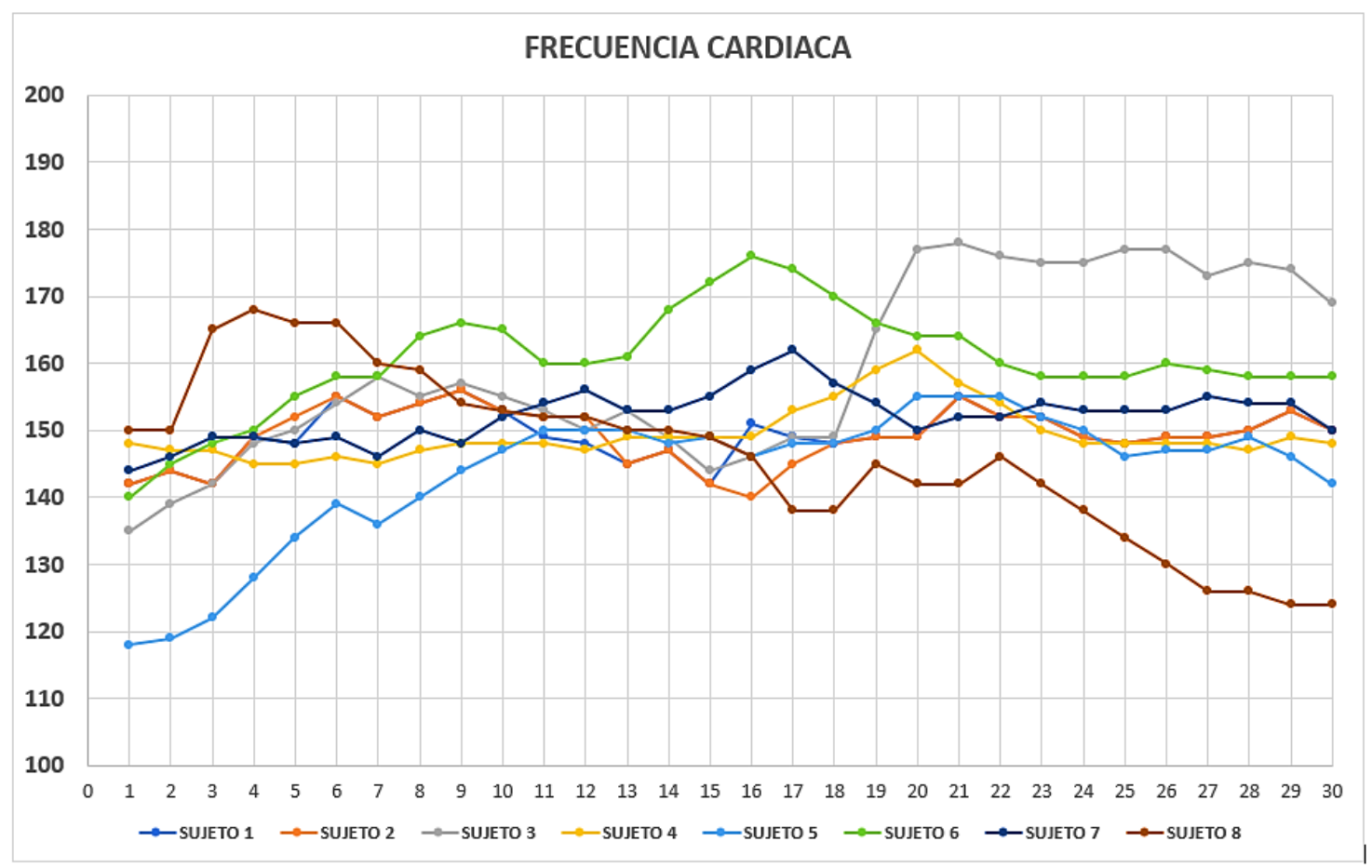

\section{Figura 5}

Registro de la temperatura corporal $\left({ }^{\circ} \mathrm{C}\right)(Y)$ de cada árbitro participante en las pruebas durante 30 minutos $(X)$ en un gráfico de dispersión

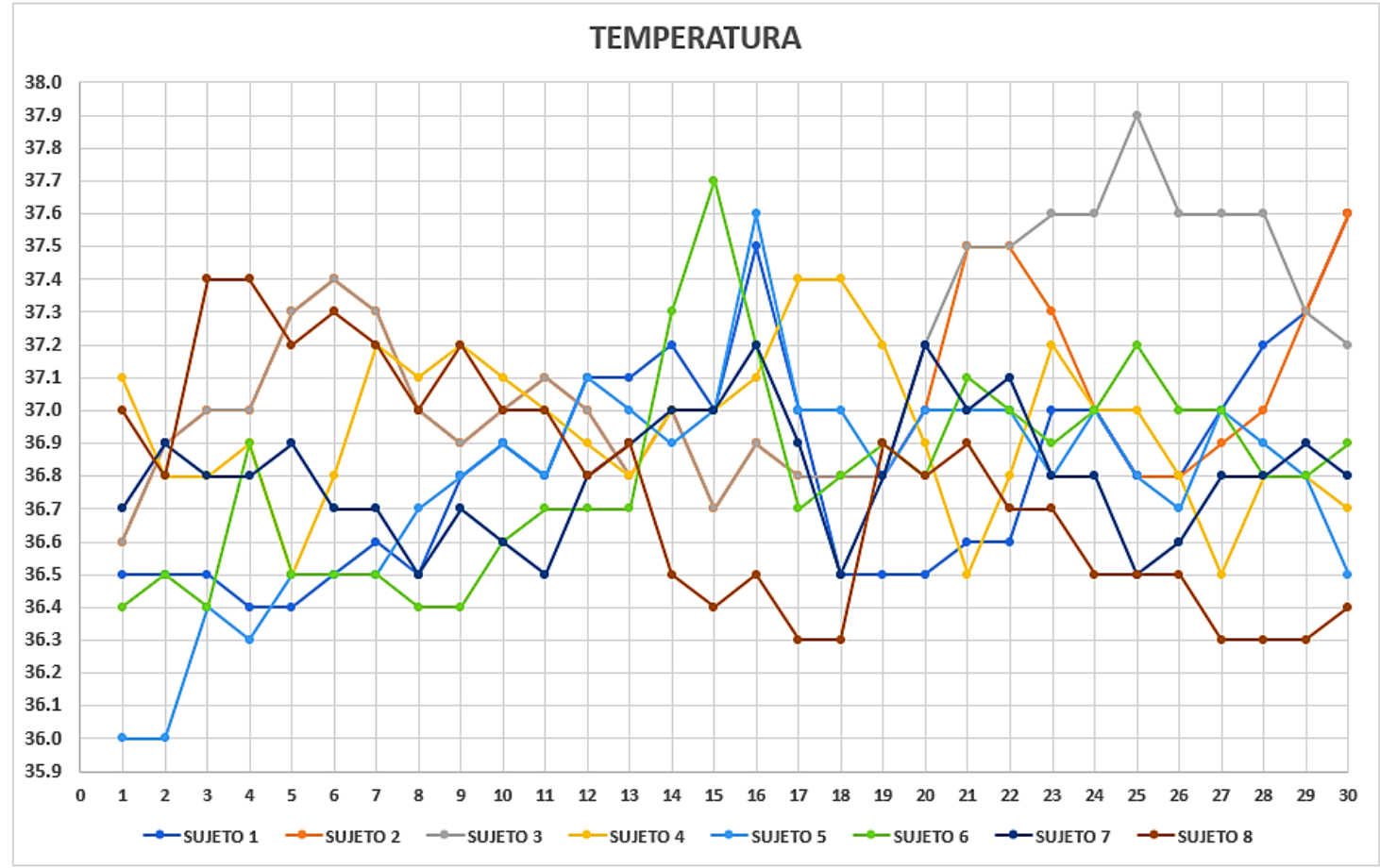




\section{Conclusiones}

Para el desarrollo de esta investigación se implementa el prototipo de sensores de frecuencia cardiaca y temperatura corporal mediante la tecnología IoT, usando una plataforma ESP32 con conectividad WIFI. Además, se desarrolla el aplicativo para la transducción de las señales biométricas en tiempo real, a valores físicos para su estudio y análisis.

Mediante pruebas de campo a los árbitros de fútbol participantes del estudio, se puede validar el funcionamiento de la herramienta utilizada, analizar los datos obtenidos y determinar el relacionamiento entre las acciones realizadas por los árbitros y sus emociones durante la toma de decisiones, con la finalidad de inferir la influencia de las emociones sobre la toma de decisiones.

Los estados emocionales pueden generar respuestas estereotipadas o automáticas ante diferentes situaciones debido a la naturaleza funcional de la misma. Pero tal y como se observa en los resultados de esta investigación, el comportamiento emocional es más concreto al realizar una tarea para la cual, el individuo ha sido formado o ha generado horas de entrenamiento y puesta en práctica de dicha conducta. Esta conducta requiere el análisis de una situación para emitir un veredicto de manera casi inmediata y genera la no interferencia del estado emocional al menos en un nivel no tan decisivo.

Mediante la diferente bibliografía consultada se puedo instaurar, la variación de la frecuencia cardiaca y temperatura como parámetros fisiológicos para la identificación de los rangos que se establecieron para cada uno de los estados en la aplicación de las pruebas a los árbitros participantes. No se puedo hallar en estas bases bibliográficas relación entre los datos biométricos musculares tomados por el sensor electromiográfico (parte del prototipo), con las emociones.

Los sensores de registro de las variables fisiológicas tienen una adecuada sinergia permitiendo la toma correcta de las variables y su posterior tratamiento en el programa desarrollado en el aplicativo LabVIEW, permitiendo observar en tiempo real la recolección exitosa de los datos para ser analizados con las acciones arbitrales en el transcurso de las pruebas realizadas. No obstante, se consideran las características de margen de error, precisión y sensibilidad de los sensores utilizados en el prototipo, realizando la comparación y validación con dispositivos homologados.

El análisis de las pruebas de campo se realizaron con el criterio y seguimiento de un experto en el área (ex árbitro profesional), a quien se le mostró el material audiovisual de las pruebas realizadas para el vídeo análisis. El experto observó que los árbitros no tomaron decisiones que se encontraran fuera del reglamento deportivo, relacionando el criterio del experto sobre las acciones de los árbitros en este periodo, con los datos registrados por el prototipo. Con ello, se logró determinar que durante estas pruebas la labor profesional de dichos árbitros no fue influenciada a pesar de la presencia de los diferentes estados emocionales, así como su correcto desempeño arbitral. Durante el desarrollo de estos encuentros deportivos, los sujetos puestos a prueba lograron el manejo y control de sus emociones, realizando su labor con objetividad y profesionalismo.

Se debe considerar aumentar número de participantes en las pruebas con la finalidad de generar análisis paramétricos que permitan comprobar la validez de la hipótesis, es decir, si realmente los estados emocionales afectan o no en las decisiones arbitrales y generan una línea base de desempeño atlético con el objetivo de tomar las variaciones en las medidas fisiológicas 
para identificar que son a causa de un cambio en el estado emocional y no por el rendimiento físico.

Para este proyecto, se identifica en las pruebas la mayor cantidad de registros por frecuencia en el estado emocional 4 (cuadrante placer-desactivación), donde la frecuencia cardiaca está por debajo de la media. Se asume que debido a que fueron tomadas en partidos amistosos y el participante logra dirigir con tranquilidad el encuentro, por tal motivo, se recomienda realizar pruebas en partidos de ligas profesionales, ya que, al ser encuentros de nivel, se considera que infiere mayor presión y estrés.

Además, para una mejora del prototipo de sensores y el análisis de futuras investigaciones, se puede implementar como herramienta de toma de datos, sensores de conductividad eléctrica de la piel (GSR), opción relevante para este tipo de estudios.

\section{Referencias}

Aguilar-Barojas, S. (2005). Fórmulas para el cálculo de la muestra en investigaciones de salud. Salud en Tabasco, 11(1-2), 333-338.

Castillo, D., Cámara, J., y Yanci, J. (2016). Análisis de las respuestas físicas y fisiológicas de árbitros y árbitros asistentes de fútbol durante partidos oficiales de Tercera División de España. RICYDE. Revista Internacional de Ciencias del Deporte, XII(45), 250-261.

Cisneros, M., Malagon, A., y Redondo, J. P. (2013). Desarrollo de técnicas de detección automática de estado de ánimo mediante redes de sensores inalámbricas. Madrid: UNIVERSIDAD COMPLUTENSE DE MADRID, Proyecto de Sistemas Informáticos.

Escobar, D. P., Navarro, W. H., Medavilla, C. M., y León, D. A. (2020). La preparación física en la toma de decisiones arbitrales del fútbol. Polo del Conocimiento: Revista científico-profesional,, 3-11.

Fava, L., Vilches, D., Ferraresso, A., Boccalari, E., y Díaz, J. (2020). Inteligencia y tecnologías aplicadas al deporte de alto rendimiento. Red de Universidades con Carreras en Informática (RedUNCI), 698-702.

Fonseca Mora, L. E. (2017). El desarrollo emocional en el desempeño conductual de los árbitros profesionales de fútbol del cantón Ambato. Ambato: Universidad Técnica de Ambato. Facultad de Ciencias Humanas y de la Educación. Maestría en Cultura Física y Entrenamiento Deportivo.

Maier, A., Sharp, A., y Vagapov, Y. (2017). Comparative analysis and practical implementation of the ESP32 microcontroller module for the internet of things. Internet Technologies and Applications (ITA), 143148.

Mallo, J., García, J. M., y Navarro, A. E. (2007). Evaluación del rendimiento físico de los árbitros y árbitros asistentes durante la competición en el fútbol. Archivos de medicina del deporte. revista de la Federación Española de Medicina del Deporte y de la Confederación Iberoamericana de Medicina del Deporte, XXIV(118), 91-102.

MedlinePlus. (7 de 2019). https://medlineplus.gov/spanish/. (Biblioteca Nacional de Medicina de EE. UU.)

Ortega, A., Villamizar, D., y Zahir, R. (2014). Toma de decisiones en árbitros de futbol: una mirada hacia la eficacia y eficiencia. Actividad fisica y desarrollo humano.

Ortiz, A. D. (2010). Teoría de las emociones. Innovación y experiencias educativas(29).

Vindel, A. C. (2010). Ansiedad, estrés, emociones negativas y salud. Atrapados por el estrés. Crítica(968), 1216.

Watson, D., y Tellegen, A. (1985). Toward a consensual structure of mood. Psychological Bulletin, 219-335. 
Yik, M., Russell, J., y Feldman Barrett, L. (1999). Structure of self reported current affect: Integration and beyond. Journal of Personality and Social Psychology, 77, 600-619. 